\title{
Práticas e crenças maternas sobre cuidado e estimulação de bebês prematuros e a termo
}

\author{
Olga Maria Piazentin Rolim Rodrigues* \\ Bárbara Camila de Campos** \\ Jussânia Moraes Martins*** \\ Flávia Helena Pereira Padovani**** \\ Projeto financiado pela FAPESP E CAPES
}

\begin{abstract}
Resumo
O objetivo do estudo foi descrever e comparar práticas parentais e crenças sobre desenvolvimento de 23 mães de bebês nascidos prematuros de muito baixo peso e de 23 mães de bebês nascidos a termo. Os dados sociodemográficos foram coletados em uma entrevista inicial e foi aplicada a "Escala de crenças parentais e práticas de cuidado na primeira infância” (E-CPPC). Os dados obtidos indicaram que na dimensão Cuidados Primários não houve diferença estatisticamente significativa entre os grupos quanto às práticas, mas houve diferenças considerando as crenças. Para a dimensão Estimulação, as mães de bebês prematuros a realizam em maior frequência do que as mães dos bebês a termo, mas acham menos importante fazê-las. Ou seja, mostram-se informadas a respeito de cuidado e estimulação e como fazê-la, entretanto, parece que não entenderam a sua importância. Os dados sugerem que no serviço de intervenção precoce aprendem a estimular seu bebê, mas não porque fazê-lo.

Palavras-chave: Recém-Nascido Prematuro, Crenças Parentais, Práticas de Cuidado.

Maternal practices and beliefs about primary care and stimulation of preterm and term babies

\section{Abstract}

The aim of the study was to describe and compare practices and parental beliefs about development of 23 mothers of premature babies with very low weight and 23 mothers of term infants. Sociodemographic data were collected in an initial interview and the "Parental beliefs and care practices in early childhood" scale (E-CPPC) was applied. The data indicated that in the Primary Care dimension there was no statistically significant difference between the groups regarding the practices, but there were differences considering the beliefs. For the Stimulation dimension, mothers of preterm infants perform it more frequently than the mothers of full-term babies, but find it less important to do so. That is, they are informed about care and stimulation and how to do it, however, it seems that they did not understand its importance. Os dados sugerem que no serviço de intervenção precoce aprendem a estimular seu bebê, mas não porque fazê-lo.
\end{abstract}

Keywords: Newborn Premature, Parenting beliefs, Caring practices.

* Livre Docente do Departamento de Psicologia - Universidade Estadual Júlio de Mesquita Filho - UNESP - Bauru (SP) - Brasil

** Doutoranda do Programa de Pós Graduação em Psicologia do Desenvolvimento e Aprendizagem, da Universidade Estadual Júlio de Mesquita Filho UNESP - Bauru (SP) - Brasil

***Mestre em Psicologia do Desenvolvimento e Aprendizagem, Universidade Estadual Júlio de Mesquita Filho - UNESP - Bauru (SP) - Brasil

****Professora Assistente Doutora do Departamento de Neurologia, Psicologia e Psiquiatria da Faculdade de Medicina de Botucatu - UNESP Universidade Estadual Júlio de Mesquita Filho - UNESP - Botucatu (SP) - Brasil 


\section{Introdução}

O desenvolvimento durante os primeiros anos de vida é permeado por importantes mudanças nas estruturas físicas, neurológicas e comportamentais (Gaíva \& Scochi, 2005). Estudos sobre essa fase identificaram que a exposição à fatores de riscos biológicos e ambientais estão relacionados ao aumento da probabilidade de atraso ou comprometimento no crescimento e desenvolvimento infantil (Gaíva, \& Scochi, 2005; Bolsoni-Silva, Marturano \& Loureiro 2009; Bueno, Castro \& Chiquetti, 2014; Campos \& Rodrigues, 2015; Dessen, 1995; Keller, 2002). Dentre os fatores citados pelos autores estão a má formação congênita, baixo peso ao nascimento, complicações no parto e na gravidez, prematuridade, baixa condição socioeconômica, saúde emocional materna, práticas parentais negativas e a separação conjugal como importantes variáveis de risco.

Segundo a Organização Mundial da Saúde, o Brasil é o décimo país com nascimentos pré-termo no mundo com uma prevalência estimada de 9,2\% (WHO, 2012). Associado à prematuridade os bebês podem apresentar danos clínicos e neurológicos interferindo em sua sobrevida, qualidade e condições de vida no primeiro ano após o nascimento, devido a imaturidade do organismo como um todo (Figueiredo, Dias, Brandao, Canario \& Nunes-Costa, 2013). Mesmo os que conseguem superar o período neonatal de risco podem apresentar alterações no desenvolvimento e saúde (Bueno, Castro, \& Chiquetti, 2014) e dificuldades nas áreas cognitivas, motoras e de linguagem, vivenciando atrasos na aquisição de alguns comportamentos nos anos iniciais (Keller, 2002; Keller, Dias, Brandao, Canario \& Nunes-Costa, 2007; Kobarg \& Vieira, 2008).

O avanço na área de neonatologia, nas últimas décadas, ampliou a sobrevida dessa população, entretanto, o prognóstico aponta para maior vulnerabilidade e risco para desenvolvimento atípico. Linhares, Carvalho, Machado e Martinez (2003) ressaltaram a possibilidade de que o atraso presente no primeiro ano de vida seja, mais tarde, recuperado. Neste caso, um importante fator de proteção é a família, enquanto primeiro contexto de desenvolvimento humano, exercendo um papel fundamental na aquisição e manutenção de novos repertórios comportamentais (Maia \& Williams, 2005; Braz, Dessen \& Silva, 2005).

Por ocasião do nascimento prematuro os bebês podem permanecer um período em Unidades de Terapia Intensivas Neonatais (UTIN). É uma nova realidade que requer adaptação e cuidados diferenciados da família, pois durante a internação há pouca participação dela nos cuidados e possibilidade de interações com o bebê (Ramos, Lima, Leal, Prado, Oliveira, Souza \& Leite, 2016). Um estudo conduzido por Ribeiro, Perosa e Padovani (2014) indicou que mães de bebês nestas condições tendem a apresentar mais sentimentos de incerteza, apreensão, tristeza, separação precoce e culpa, diante dessa realidade de pós-parto atípica e potencialmente estressora. Investigações após a alta hospitalar conduzidas por Piccinni, Frizzo, Brys e Lopes (2014) mostraram que mães de bebês prematuros foram menos responsivas, mais intrusivas, hostis ou apresentaram retraimento em relação ao bebê ocasionando um ambiente com interações mais restritas.

Em relação às concepções maternas sobre o cuidado e a estimulação oferecida a bebês prematuros os achados indicaram que, de maneira geral, as mães se preocupam em oferecer condições relativas ao seu bem estar físico e de desenvolvimento motor global e dão menor importância aos comportamentos de estimulação, questões sociais e a disciplina (Cossul, Silveira, Pontes, Martins, Wernet, \& Cabral, 2015; Guardiano, Passas, Corujeira, Gonçalves, Almeida, \& Viana, 2017; Lopes, Silva, Rocha, Avelino, \& Soares, 2015).

A maneira de cuidar e estimular as crianças sofre múltiplas influências, podendo variar conforme o ambiente, a cultura e a história da díade (Keller, 2002; Braz, Dessen \& Silva, 2005, Targino \& Bandeira, 2012). Analisar como os cuidadores se comportam, sob o ponto de vista teórico e prático (ou seja, o que eles fazem e o que eles acham importante fazer) em relação as práticas promotoras de desenvolvimento dão boas pistas sobre a qualidade ou necessidades da interação entre indivíduo e o contexto.

O "Modelo de Componentes de Parentalidade" proposto por Keller et al. (2007) conta com seis sistemas parentais e quatro mecanismos moduladores da interação de características universais, com comportamentos semelhantes em todas as culturas, sendo eles os de: cuidado primário, contato corporal, estimulação corporal, estimulação por objetos, contato face-a-face, linguagem e narrativa, esses comportamentos e a sincronia entre a díade diante da expressão desses é modulada por quatro estruturas: atenção, calor emocional, contingência e responsividade à expressão de emoções. Baseados neste modelo, Martins, Macarini, Vieira, Seidl-de-Moura, Bussab \& Cruz (2010) reuniram evidências de validade fatorial para a construção e validação da "Escala de Crenças Parentais e Práticas de Cuidado (E-CPPC) na primeira infância". Para a validação os autores utilizaram amostras 
de 250 e 600 mães e identificaram dois fatores: cuidados primários e estimulação com Alpha de Cronbach de 0,68 e 0,83 indicando estimativa de confiabilidade que permitem mensurar as práticas parentais de mães no contexto brasileiro. A escala conta com 25 comportamentos reunindo fatores apresentados em duas dimensões em que devem assinalar a frequência das "Práticas Realizadas pela Mãe" (PRM) e, em seguida, registrar a "Importância Atribuída às Práticas" (IAP) para o desenvolvimento infantil.

Cossul et al.(2015) realizaram um estudo com o objetivo de identificar as práticas e crenças parentais adotadas no cuidado à criança prematura no domicílio. Participaram 31 pais que responderam a ficha da família e a "Escala de crenças parentais e práticas de cuidado (E-CPPC)". Os resultados apontaram para diferenças significativas entre as crenças e as práticas, tanto de cuidado quanto de estimulação, com médias maiores para as crenças. As autoras concluem que os pais acham importante fazer (cuidar e estimular o bebê prematuro), mas fazem pouco.

Um estudo foi realizado por Campos e Rodrigues (2015) para descrever e relacionar o índice de depressão pós-parto apresentado por mães de bebês e as práticas e crenças sobre cuidado primário e estimulação. Foi utilizada a "Escala de Edinburgh de Depressão Pós-Parto - EPDS" e a "Escala de crenças parentais e práticas de cuidado (E-CPPC) na primeira infância". Os resultados obtidos com 132 mães indicaram sintomas de depressão para $29,5 \%$ da amostra. Com relação às práticas houve diferenças significativas entre os grupos clínicos e não clínicos na dimensão Estimulação, indicando que mães deprimidas interagem e estimulam menos seus bebês. As autoras concluem a díade deve ser cuidada para garantir o desenvolvimento adequado dos bebês.

Minetto e Löhr (2016) utilizaram a Escala de Crenças Parentais e Práticas de Cuidado (E-CPPC) com 75 mães de crianças, sendo 41 crianças com diagnóstico de deficiência intelectual (DI) e 34 com Síndrome de Down (SD). Os resultados apontaram que mães de crianças com SD valorizaram mais a estimulação do que as mães de crianças com DI que valorizaram mais a prática de cuidados. As autoras atribuem à identificação precoce da síndrome e a busca mais rápida de serviços de apoio.

Correa, Minetto, Cappellaro-Kobren e Kruszielski (2018) investigaram as crenças e as práticas parentais de estimulação e cuidados utilizando a E-CPPC em 36 famílias de crianças com atrasos no desenvolvimento. Entre os resultados, verificou-se que quanto maior a escolaridade, menor número de pessoas na residência e pais em união estável, maior a importância dada à estimulação da crian- ça. Os autores também observaram que quanto maior a frequência à uma religião, maiores as práticas de cuidado.

Martins et al.(2010) avaliaram a influência do contexto nas práticas e crenças parentais, a partir da E-CPPC, comparando famílias nascidas em grandes centros com de cidades pequenas. De maneira geral estas pesquisas indicam que as mães relataram realizar e atribuíram maior importância aos cuidados primários (alimentação, higiene, abrigo, segurança e proximidade corporal da díade) em ambos contextos. Os resultados indicaram que as práticas de estimulação foram realizadas e mais valorizadas pelas mães das capitais. Os dados sugerem que à medida que as famílias passam a ter acesso à educação formal, são mais expostas a informações sobre práticas parentais e sobre o desenvolvimento de suas crianças determinando valores e crenças sobre como os filhos devem ser educados.

As concepções maternas sobre cuidar e estimular crianças acompanham as características sociais e culturais decorrente de cada momento histórico. Neste sentido, as condições de assistência hospitalar intensiva oferecidas aos bebês pré-termo podem gerar mudanças nas dinâmicas familiares. Torna-se relevante, então, a investigação dos sistemas de cuidado à criança, com pesquisas sobre como é a articulação entre crenças e práticas do cuidador levando em conta bebês que nasceram pré-termo e a termo como forma de avaliar se há alguma implicação da prematuridade na responsividade da mãe à criança.

\section{Objetivo:}

O presente projeto pretendeu descrever e comparar as práticas de cuidados e de estimulação de mães de bebês prematuros e nascidos a termo.

\section{Método}

\section{Participantes:}

Participaram da amostra 56 crianças, de ambos os sexos, na faixa etária de 22 a 43 meses, distribuídas em dois grupos. O Grupo Pré Termo (GPT) foi formado por 23 crianças nascidas pré-termo que faziam acompanhamento no Ambulatório de Follow-Up da UTI Neonatal, de um hospital universitário do interior paulista, cujas mães e/ou cuidadoras aceitaram participar do estudo. O Grupo a Termo (GAT), por sua vez, foi formado por 23 crianças a termo que eram atendidas no Centro de Saúde Escola (CSE), cujas mães e/ou cuidadoras aceitaram participar da pesquisa. Foram excluídas da amostra crianças com suspeita ou com alterações neurológicas, genéticas ou metabólicas. 
A idade média das 56 mães entrevistadas foi de 29,6 (DP=10,61) anos no GPT e de 32,3 (DP= 5,47) no GAT. Em relação à realização de atividade remunerada a porcentagem de mães do GAT foi maior $(60,8 \%)$, quando comparadas ao GPT (35,9\%), entretanto não houve diferença estatisticamente significativa entre os grupos nessas variáveis. Quanto à escolaridade a das mães do grupo de prematuros variou entre Ensino Fundamental e Médio $(28,5 \% ; 35,7 \%)$ e as mães de bebês a termo tiveram maior concentração na categoria Ensino Médio $(60,7 \%)$. Nesta categoria, considerando a média de anos estudados houve diferença estatística $(p=0,050)$, com média mais alta para as mães do GAT com oito anos de estudo e seis para as do GPT.

A idade gestacional e peso médio do GPT foram de $27(\mathrm{DP}=2,8)$ semanas e 990,5g $(\mathrm{DP}=282,7)$, para o GAT foram em média $38(\mathrm{DP}=5,5)$ semanas e $3275 \mathrm{~kg}$ (DP=661,5). Considerando o sexo dos bebês não houve diferença estatisticamente significativa entre os grupos assim como para a idade do bebê na avaliação, com média de 29,6 meses para os bebês prematuros e 32,1 meses nos a termo $(\mathrm{p}=0,111)$.

\section{Instrumentos:}

Para coleta dos dados sociodemográficos e sobre a saúde do bebê foi elaborada uma Entrevista Inicial para a mãe (ou cuidadora). Para os bebês do GTP os dados foram obtidos do prontuário médico da criança, que registra sua história enquanto paciente do hospital, para obtenção de dados sobre as condições de nascimento, hospitalização e possíveis comorbidades.

Os cuidados com o bebê foram avaliados a partir da "Escala de crenças parentais e práticas de cuidado (E-CPPC) na primeira infância" validada por Martins et al. (2010), previsto para crianças de zero a seis anos de idade. $\mathrm{O}$ instrumento consiste em 36 itens, que descrevem comportamento maternos em duas subescalas: "Práticas Realizadas pela Mãe" (PRM) e "Importância Atribuída às Práticas" (IAP), em uma escala likert. Para cada assertiva, a mãe deve assinalar a importância que atribui ao item, numa escala de cinco pontos (1="não concordo"; $5=$ "é extremamente importante”). Entre as questões há dois diferentes domínios do cuidado parental: (a) Estimulação: relacionada com práticas que estimulem o desenvolvimento cognitivo e motor da criança, como, por exemplo, oferecer brinquedos que estimulem seus sentidos e ler histórias para a criança; (b) Cuidados: relacionada com práticas orientadas para boa higiene da criança em lugares públicos e ênfase nos comportamentos socialmente aceitáveis, como manter a criança limpa e adequadamente arrumada. Os mesmos devem ser analisados em duas dimensões: a) o que realiza com o bebê e b) o que acha importante realizar com ele.

\section{Local da coleta:}

A coleta de dados do GPT foi realizada no Ambulatório de Follow-up da UTI Neonatal, do Hospital Universitário, vinculado à Faculdade de Medicina de Botucatu, da UNESP. O Ambulatório tem por objetivo avaliar e acompanhar o desenvolvimento das crianças nascidas pré-termo até a idade de 14 anos para intervir preventivamente no caso de identificação de risco, mediante orientações às mães (ou cuidadores) e encaminhamentos, se necessário, a outros serviços. Neste Ambulatório são acompanhadas, após alta hospitalar, todas as crianças que ficaram internadas na UTI Neonatal e, entre elas, as crianças nascidas com muito baixo peso $(\leq 1500 \mathrm{~g})$ e de prematuridade extrema que, além da avaliação médica, passam sistematicamente pela avaliação do desenvolvimento neuropsicomotor. Concomitantemente, os pais são orientados quanto à estimulação da linguagem e motricidade.

As coletas do GAT, por sua vez, foram realizadas nas instalações das Unidades de Atenção Primária da Família, Centro de Saúde Escola - Unidade Auxiliar da Faculdade de Medicina de Botucatu - Vila dos Lavradores (CSE/ FMB - UVL). As Unidades de Atenção Primária trabalham com um conjunto de ações com o objetivo de realizar a profilaxia de doenças na população, facilitando na remoção dos fatores causais, minimizando a incidência de doenças.

\section{Procedimentos:}

A pesquisa foi aprovada pelo Comitê de Ética Faculdade de Medicina de Botucatu (Processo no 265.306). As participantes, após esclarecimentos quanto à sua participação na pesquisa e concordância quantos aos termos dela, assinavam um Termo de Consentimento Livre e Esclarecido. Em seguida eram convidadas a responder a Entrevista Inicial e a E-CPPC. Para o GPT a coleta de dados foi realizada durante consulta de rotina no Ambulatório de Follow-Up da UTI Neonatal da UNESP e, para o GAT, a avaliação ocorreu na Unidade de Saúde, quando elas compareciam para consultas de Puericultura.

\section{Análise de Dados:}

Os dados da "Escala de Crenças Parentais e Práticas de Cuidado (E-CPPC) na primeira infância" foram obtidos a partir da soma dos valores absolutos em cada domínio, denominados Práticas (Faz) e Crenças (Acha 
importante), divididos pelo número de itens em cada uma das dimensões: Cuidados Primários e Estimulação. Para este estudo elaborou-se critérios arbitrários para a avaliação comparativa das práticas e crenças relatadas pelas mães dos dois grupos. Definiu-se como pontuação abaixo da média 32 a 34 pontos, na média, de 35 a 37 pontos e, de 38 a 40 pontos, acima da média. Os comportamentos relativos aos Cuidados são: socorrer quando está chorando; alimentar; manter limpa; cuidar para que durma e descanse; não deixar que passe frio ou calor; carregar no colo; ter sempre por perto; tentar evitar que se acidente (cuidados de segurança). Os relativos à Estimulação são: deixar livre para correr, nadar, trepar; brincadeiras de luta, de se embolar (corporais); fazer atividades físicas; jogar jogos; ver livrinhos juntos; mostrar coisas interessantes; explicar coisas; ouvir o que tem a dizer, responder perguntas e, ficar frente a frente, olho no olho.

Para realizar os testes estatísticos dos dados obtidos nesta entrevista e na escala foi utilizado o SPSS (Statistical Package for Social Sciences 21.0) e, os dados foram analisados pelo teste T de Student, com o objetivo de verificar se havia diferenças significativas (intervalo de confiança de $95 \% ; \mathrm{p}=0,05)$ entre os grupos considerando a frequência de realização dos comportamentos, tanto geral quanto em amostras do GPT e GAT.

\section{Resultados}

Os dados inicialmente se referem àqueles obtidos com a "Escala de crenças parentais e práticas de cuidado (E-CPPC) na primeira infância”. Foram feitas comparações inter e intragrupos, considerando-se as frequências absolutas e relativas.

A Tabela 1 apresenta os resultados da comparação entre os grupos considerando a pontuação média obtida na dimensão de Cuidados. Análises estatísticas apontaram para diferença significativa para as crenças relacionadas aos cuidados $(\mathrm{p}=0,008)$, com média maior para as mães do GAT. Comparando, cada grupo para a dimensão de Cuidados em relação às Práticas e Crenças, observa-se que houve diferenças significativas entre as duas dimensões sendo que nos dois grupos a média foi significativamente maior para a prática $(\mathrm{GPT}=36,92 ; \mathrm{GAT}=37,21)$ do que para crenças $(\mathrm{GPT}=35,78$; GAT $=36,5)$.

A Tabela 2 apresenta os resultados da comparação entre grupos considerando a pontuação média obtida na escala, em Estimulação. Análises estatísticas apontaram para diferença significativa para as Crenças relacionadas à Estimulação $(p=0,000)$, com melhor desempenho para as mães do GAT. Comparando, cada grupo para a dimensão de Estimulação em relação às Práticas e Crenças, observa-se que houve diferenças significativas entre as duas dimensões sendo que nos dois grupos a média foi significativamente maior para a Crença (GPT=39,17; GAT=41,89) do que para a prática $(\mathrm{GPT}=35,78 ; \mathrm{GAT}=36,5)$.

Tabela 1. Comparação da pontuação média dos participantes dos grupos em Cuidados, nas dimensões práticas e crenças.

\begin{tabular}{llllll}
\hline Cuidado & Prática & & Crenças & & P \\
\hline & Média & DP & Média & DP & \\
GPT & 36,92 & 2,1 & 35,78 & 2,9 & 0,000 \\
GAT & 37,21 & 2,4 & 36,5 & 3,5 & 0,019 \\
P & 0,133 & & 0,008 & & \\
\hline
\end{tabular}

Tabela 2. Comparação da pontuação média dos participantes dos grupos em Estimulação, nas dimensões práticas e crenças.

\begin{tabular}{llllll}
\hline Estimulação & Prática & \multicolumn{3}{c}{ Crenças } & P \\
\hline & Média & DP & Média & DP & \\
GPT & 35,78 & 2,9 & 39,17 & 4,78 & 0,000 \\
GAT & 36.5 & 3,53 & 41,89 & 4,55 & 0,001 \\
P & 0,404 & & 0,000 & & \\
\hline
\end{tabular}

Análises foram feitas associando os comportamentos maternos de práticas e crenças entre Cuidado e Estimulação, para cada um dos grupos. Os resultados da Tabela 3 mostraram os dados obtidos para o GPT. Observou-se correlação positiva entre as práticas e as crenças de Cuidado $(\mathrm{p}=0,000)$ mas não entre as práticas e as crenças de Estimulação $(p=0,173)$.

Tabela 3. Correlação entre práticas e crenças, do GPT.

\begin{tabular}{lll}
\hline Correlação dos indicadores & Rho & P \\
\hline Cuidado prática X cuidado crenças &, $973^{* *}$ &, 000 \\
Estimulação prática X estimulação crenças &, 265 & 0,173 \\
\hline
\end{tabular}

$\mathrm{Na}$ Tabela 4 estão os dados da análise das associações entre práticas e crenças, de Cuidado e de Estimulação para o GAT. As correlações foram positivas tanto na dimensão de Cuidados quanto na de Estimulação. A associação entre as práticas e crenças de Cuidado foi significativa $(p=0,000)$, mas não em práticas e crenças em Estimulação $(p=0,052)$. 
Tabela 4. Correlação entre práticas e crenças, nos aspectos Cuidados e Estimulação, do GAT.

\begin{tabular}{lll}
\hline Correlação dos indicadores & Rho & P \\
\hline Cuidado práticas X cuidado crenças &, $940 * *$ &, 000 \\
Estimulação práticas X estimulação crenças &, 372 &, 052 \\
\hline
\end{tabular}

\section{Discussão}

O presente trabalho pretendeu descrever e comparar as práticas e crenças de mães sobre Cuidados e Estimulação oferecidos aos seus bebês considerando a condição de nascimento como variável independente, crianças nascidas prematuras e à termo. Os resultados apontaram algumas diferenças importantes entre os grupos. Considerando o total de pontos obtidos na E-CPPC, mães do GAT relataram, em maior frequência, realizar práticas e compartilhar crenças de cuidados do que as mães do GPT.

Observando os dados obtidos sobre as práticas de mães de bebês relacionadas a Cuidado as análises evidenciaram que não houve diferença estatisticamente significativa entre os grupos. Além disso, quando cada grupo foi comparado separadamente, observou-se que as mães dos dois grupos também pontuaram mais na realização dos cuidados do que acreditam que seja importante, ou seja, independente da condição (prematuros ou a termo) emitir em maior frequência comportamentos dessa classe é uma característica cultural observada em outros estudos (Martins et al., 2010; Pasquali, Gouveia, Santos, Fonseca, Andrade \& Lima, 2012; Piccinni, Frizzo, Brys \& Lopes, 2014; Plutarco Viana, Santos, De Andrade, Nádia, \& Lopes, 2014).

Neste sentido os dados sobre o Cuidado e Estimulação indicaram um importante dado cultural de mães do sudeste brasileiro: elas tendem a investir mais em práticas de cuidados primários com seus bebês. Esse tipo de prática envolve comportamentos com características de cuidado básico e físico que são típicos de aprendizagens por regras de disciplina típicas de práticas populares (Lopes et al., 2015; Souza \& Ferreira, 2010; Braz, Dessen \& Silva, 2005; Ramos et al., 2016).

Com relação à Estimulação, as mães de crianças nascidas prematuras realizam este comportamento mais do que as mães de crianças nascidas a termo. Contudo, essas mães não assinalaram na mesma frequência a dimensão sobre a importância dessa prática. Levando em conta que essas mulheres participam de um serviço de acompanhamento aos seus bebês desde a alta hospitalar, este dado parece indicar que o serviço a que seus bebês estão expostos é uma boa rede de apoio, pois é importante que esses bebês sejam estimulados, entretanto há a possibilidade de que as orientações sejam oferecidas de maneira prescritiva, ou seja, dizem a mãe o que devem fazer, mas não porque devem fazer. O estudo de Lopes $e t$ al. (2015) sobre amamentação em prematuros identificou que o relato das mães sobre amamentação e conhecimento teórico era adequado, entretanto ao observarem essas mães amamentando o relato estava em descompasso com a qualidade da prática. Neste sentido os dados indicam a importância de uma equipe de saúde atenta a qualidade e ao grau de compreensão das informações por parte da família ( Gaíva \& Scochi, 2005; Ramos et al., 2016; Souza \& Ferreira, 2010).

\section{Considerações finais}

A necessidade de se investigar as práticas parentais de cuidado e de estimulação desde as fases iniciais do desenvolvimento foi apontada por Keller (2002), pois os resultados obtidos poderão embasar intervenções precoces envolvendo a família. $\mathrm{Na}$ presente pesquisa os dados confirmam uma tendência de outros estudos, que mostraram que as mães privilegiam os cuidados tanto no que fazem quanto na importância que atribuem a ele. Também, indicaram que mães de bebês prematuros tendem a se comportar de maneira diferente quanto a qualidade e quantidade de estimulação oferecida aos seus bebês, os estimulando mais do que as mães de bebês nascidos a termo.

Um dado importante se refere à pouca importância que as mães de prematuros atribuem à estimulação de seus bebês, apesar de oferecê-la mais do que as mães dos bebês a termo. Os dados sugerem a hipótese de que a maneira como as informações sobre a necessidade de estimulação chega aos pais pode ser responsável por esses dados. Este estudo sugere que a maneira que a equipe organiza e oferece as orientações seja revista, pois, apesar de estimular seu filho permanece a crença de que não é importante fazê-lo.

Porém, tais dados precisam ser confirmados com populações maiores e mais homogêneas em algumas das características demográficas como, por exemplo, a escolaridade, considerando que entre os limites deste estudo incluem o tamanho e perfil diferente das amostras de cada grupo. Os resultados obtidos sugerem novas pesquisas que podem ser conduzidas com populações maiores de prematuros comparando com grupo controle pareados por características sociodemográficas, assim como pesquisas com populações com características socioculturais diferentes, como forma de aferir o peso delas nas crenças sobre práticas de cuidados e de estimulação de bebês. Um 
outro ponto a ser pesquisado seria a análise de crenças de pais e mães de bebês, comparando-os.

\section{Referências}

Bolsoni-Silva, A.T., Marturano, E. M., \& Loureiro, S. R. (2009). Construction and validation of the brazilian questionário de respostas socialmente habilidosas segundo relato de professores (QRSH-PR). Spanish Journal of Psychology, 12(1), 349-359. https://doi.org/10.1017/S1138741600001748

Bueno, E. A., de Castro, A. A. M., \& Chiquetti, E. M. dos S. (2014). Influência do ambiente domiciliar no desenvolvimento motor de lactentes nascidos pré-termo. Revista Neurociencias, 22(1), 45-52. https://doi.org/10.4181/ RNC.2014.22.914.8

Campos, B. C. D., \& Rodrigues, O. M. P. R. (2015). Depressão Pós-Parto Materna : Crenças, Práticas de Cuidado e Estimulação de Bebês no Primeiro Ano de Vida. Psico (Porto Alegre), 46(4), 483-492. https://doi. org/10.15448/1980-8623.2015.4.20802

Correa, W.; Minetto, M.F.; Cappellaro-Kobren, R.; Kruszielski, L. Crenças sobre práticas parentais em crianças em famílias de crianças com atraso no desenvolvimento. International Journal of Developmental and Educational Psychology, INFAD, Revista de Psicología, n. 1, v. 2, p. 21-30, 2018.

Cossul, M. U., Silveira, A. O., Pontes, T. B., Martins, G., Wernet, M., \& Cabral, C. C. de O. (2015). Parenting beliefs and practices regarding domiciliary care of premature infants. REME: Revista Mineira de Enfermagem, 19(4), 830-835. https://doi.org/10.5935/1415-2762.20150064

Dessen, M. A. (1995). Tecnologia de Video : Registro de Interacõos Sociais e Cálculos de Fidedignidade em Estudos Observacionais 1 Video Technique : On the Recording of Social Interactions and the Scoring of Reliability in Observational Studies. 11, 223-227.

Figueiredo, B., Dias, C. C., Brandao, S., Canario, C., \& Nunes-Costa, R. (2013). Breastfeeding and postpartum depression: state of the art review. Jornal de Pediatria, 89(4), 332-338. https://doi.org/10.1016/j.jped.2012.12.002

Fraga, D. A., Linhares, M. B. M., Carvalho, A. E. V., \& Martinez, F. E. (200). Desenvolvimento de bebês prematuros relacionado a variáveis neonatais e maternas. Psicologia Em Estudo, 13(2), 335-344. https://doi.org/10.1590/ S1413-73722008000200016

Gaíva, M.A.M, \& Scochi, G.S. (2005) A participação da família no cuidado ao prematuro em UTI Neonatal. Rev Bras Enferm, 58(4), 444-448. https:// doi.org/10.1590/S0034-71672005000400012

Guardiano, M., Passas, M. A., Corujeira, S., Gonçalves, D., Almeida, P., \& Viana, V. (2017). Estimulação, disciplina, vinculação e apresentação: as crenças das mães de grandes prematuros. Psic., Saúde \& Doenças, 1(18), 141-149. https://doi.org/DOI: http://dx.doi.org/10.15309/17psd180112

Keller, H. (2002). Between Culture and Biology: Perspectives on Ontogenetic Development - Heidi Keller, Ype H. Poortinga, Axel Schölmerich - Google Books. Between Culture and Biology: Perspectives on ..., 419. Retrieved from https:/ / books.google.com.br/books?hl=pt-BR\&lr=\&id=oUeDT R2MDTgC\&oi $=$ fnd\&pg $=$ PA215\&dq $=$ Development + as + the + interface + between + biology + and + culture: $+A+$ conceptualization + of + early + on togenetic + experiences\&ots $=$ Z8IWhlJP-S\&sig=OKZQzpP-ztQ7WVwHaVEpikKcm2s\#v=onepage\&q=Dev

Keller, H., Abels, M., Borke, J., Lamm, B., Lo, W., Yanjie, S., \& Yifang, W. (2007). Socialization environments of Chinese and Euro-American middle-class babies: Parenting behaviors, verbal discourses and ethnotheories. International Journal of Behavioral Development, 3(31), 210-217. Retrieved from http://journals.sagepub.com/doi/pdf/10.1177/0165025407074633

Kobarg, A. P. R., \& Vieira, M. L. (2008). Crenças e práticas de mães sobre o desenvolvimento infantil nos contextos rural e urbano. Psicologia: Reflexão E Crítica, 21(3), 401-408. https://doi.org/10.1590/S0102-79722008000300008

Linhares, M. B. M., Carvalho, A. E. V., Machado, C., \& Martinez, F. E. (2003). Desenvolvimento de bebês nascidos pré-termo no primeiro ano de vida. Paidéia (Ribeirão Preto), 13(25), 59-72. https://doi.org/10.1590/S0103$-863 \mathrm{X} 2003000200006$

Lopes, A. M., Silva, G. R. F. da, Rocha, S. S. da, Avelino, F. V. S. D., \& Soares, L. S. (2015). Amamentação em prematuros: caracterização do binômio mãe-filho e autoeficácia materna. Revista Brasileira Em Promoção Da Saúde, 28(1), 32-43. https://doi.org/10.5020/18061230.2015.p32
Maia, J. M. D., \& Williams, L. C. A. (2005). Fatores de risco e fatores de proteção ao desenvolvimento infantil: uma revisão da área. Temas Em Psicologia, 13(2), 91-103. https://doi.org/1413-389X

Martins, G. D. F., Macarini, S. M., Vieira, M. L., Seidl-de-Moura, M. L., Bussab, V. S. R., \& Cruz, R. M. (2010). Construção e validação da Escala de Crenças Parentais e Práticas de Cuidado (E-CPPC) na primeira infância. Psico-USF, 15(1), 23-34. https://doi.org/10.1590/S1413-82712010000100004

Martins, G. D. F., Vieira, M. L., Seidl-de-Moura, M. L., \& Macarini, S. M. (2011). Beliefs and care practices of mothers living in Brazilian capital cities and small towns. Psicologia: Reflexão E Crítica, 24(4), 692-701. https://doi. org/10.1590/s0102-79722011000400009

Minetto, \& Löhr. (2016). Crenças e práticas educativas de mães de crianças com desenvolvimento atipico Beliefs and educational practices of mothers of children with atypical development. 59, 49-64. https://doi.org/10.1590/0104-4060.44791

Pasquali, L., Gouveia, V. V., Santos, W. S. dos, Fons?ca, P. N. da, Andrade, J. M. de, \& Lima, T. J. S. de. (2012). Perceptions of Parents Questionnaire: Evidence for a Measure of Parenting Styles. Paidéia (Ribeirão Preto), 22(52), 155-164. https://doi.org/10.1590/S0103-863X2012000200002

Pereira, A. T., Marques, M., Marques, C., Bento, E., Azevedo, J., Xavier, S., ... Macedo, A. (n.d.). Perinatal depression screening, prevention and early intervention: recent advances in Portugal VIEWPOINT. https://doi.org/10.21035/ ijcnmh.2016.3.2

Braz, M.P. , Dessen, M. A., \& Silva, N.L.P. (2005). Relações Conjugais e Parentais: Uma Comparação entre Famílias de Classes Sociais Baixa e Média. Psicologia: Reflexão E Crítica, 18(2), 151-161. Retrieved from http://smec. salvador.ba.gov.br/site/documentos/espaco-virtual/espaco-diversidade/ RELAÇÕES DE GÊNERO/WEBARTIGOS/relacoes conjugais e parentais....pdf

Piccinni, C. A., Frizzo, G. B., Brys, I., \& Lopes, R. de C. S. (2014). Parenthood in the context of maternal depression at the end of the infant's first year of life. Estudos de Psicologia, 31(2), 203-214. https://doi.org/10.1590/0103-166X2014000200006

Plutarco Viana, T., Santos, I., De Andrade, N., Nádia, A., \& Lopes, M. (2014). Cognitive and language development in preterm infants Desenvolvimento cognitivo e linguagem em prematuros. Audiol Commun Res. R. Marcondes Pereira Joaquim Távora Lopes ANM Audiol Commun Res, 1919(781), 1-6. https://doi.org/10.1590/S2317-64312014000100002

Ramos, D. Z., Lima, C. de A., Leal, A. L. R., Prado, P. F. do, Oliveira, V. V. de, Souza, A. A. M. de, Leite, M. T. de S. (2016). A participação da família no cuidado às crianças internadas em unidade de terapia intensiva. Revista Brasileira Em Promoção Da Saúde, 29(2), 189-196. Retrieved from http:// periodicos.unifor.br/RBPS/article/view/4361/pdf

Ribeiro, D. G. [UNESP]. (2010). Desenvolvimento neuropsicomotor de crianças de um ano atendidas em unidades de ESF: fatores de risco e proteção. Aleph, 123 f. Retrieved from https://repositorio.unesp.br/ handle $/ 11449 / 98408$ ? show $=$ full

Ribeiro, D. G., Perosa, G. B., \& Padovani, F. H. P. (2014). Mental health, mother-child interaction and development at the end of the first year of life. Paidéia, 24(59), 331-339. https://doi.org/http://dx.doi.org/10.1590/198243272459201407

Rodrigues, O. M. P. R., \& Bolsoni, silva A. T. (2011). Effects of the Prematurity on the Development of Lactentes. Rev Bras Crescimento Desenvolvimento Hum, 21(14), 111-121. https://doi.org/10.7322/jhgd.20000

Souza, K. M. O. \& Ferreira, S. D. (2010). Assistência humanizada em UTI neonatal: os sentidos e as limitações identificadas pelos profissionais de saúde. Ciência \& Saúde Coletiva, 15(2), 471-480. https://doi.org/10.1590/ S1413-81232010000200024

Targino, T., \& Bandeira, A. (2012). Crenças de Pais e Mães Sobre Investimento Parental 1. 22(53), 355-363. https://doi.org/10.1590/1982-43272253201307

Wachholz, V. A., Costa, M. G., Kerber, N. P. da C., Gonçalves, C. V., Ramos, D. V., \& Sena, F. G. (2016). Relação entre a qualidade da assistência pré-natal e a prematuridade: Uma revisão integrativa. Revista Brasileira de Educação E Saúde, 6(2), 01-07. https://doi.org/10.18378/REBES.V6I2.3542

WHO. (2012). The Global Action Report on Preterm Birth Born Too Soon. Retrieved from http://www.who.int/pmnch/media/news/2012/201204_borntoosoon-report.pdf

Submetido em: 4-9-2019

Aceito em: 17-12-2019 